Check for updates

Cite this: Phys. Chem. Chem. Phys. 2021, 23, 23517

Received 26th July 2021,

Accepted 27th September 2021

DOI: $10.1039 / \mathrm{d} 1 \mathrm{cp} 03428 f$

rsc.li/pccp

\title{
Revealing the electronic properties of the B-B bond: the bis-catecholato diboron molecule $\uparrow$
}

\author{
D. Toffoli, (D) ${ }^{a}$ C. Grazioli, (D) ${ }^{b}$ M. Monti, (D) ${ }^{a}$ M. Stener, (D) ${ }^{a}$ R. Totani, (D) ${ }^{c}$ \\ R. Richter, (iD ${ }^{d}$ L. Schio, (D) ${ }^{b}$ G. Fronzoni iD *a and A. Cossaro (iD *ab
}

\begin{abstract}
The electronic properties of a diboron molecule, namely bis(catecholato)diboron (2-(1,3,2-benzodioxaborol-2-yl)-1,3,2-benzodioxaborole) $\left(\mathrm{B}_{2} \mathrm{Cat}_{2}\right)$, have been studied by comparing the results of photoemission (XPS) and near edge X-ray absorption spectroscopy (NEXAFS) experiments with the outcome of DFT calculations. The B $1 \mathrm{~s}, \mathrm{C}$ 1s and $\mathrm{O}$ 1s K-edges have been investigated for both the isolated gas phase molecule and the adsorbed one on the Au(111) surface. The main features of the polarized NEXAFS spectra at each of the three edges considered are not significantly affected by the presence of the substrate, with respect to the isolated molecule, indicating that the molecule-gold interaction is weak. Moreover, the comparison between the observed dichroism in the NEXAFS spectra of the adsorbed $\mathrm{B}_{2} \mathrm{Cat}_{2}$ and that in the NEXAFS spectra of the isolated molecule has confirmed the orbital symmetry assigned in the gas phase absorption spectra. The transitions to $\pi(B-B)$ bonding and $\pi^{\star}(B-B)$ anti-bonding final states represent the most relevant probe of the chemistry of the $B_{2} C_{2}$ molecule. We show that their theoretical description requires that the treatment of the relaxation changes among different excited state configurations, which we successfully implemented by using $\Delta$ SCF-DFT ( $\triangle \mathrm{SCF}$ ) calculations.
\end{abstract}

\section{Introduction}

Since the last two decades organo-boron chemistry has been playing an emerging role in the development of novel green and sustainable organic-based technologies. ${ }^{1-3}$ This promotes the research of convenient synthetic routes for the production of boron compounds. Among the investigated strategies, both metal-catalyzed and metal-free synthetic processes involving diboron(4) compounds as the reactants represent the most promising tool for the synthesis of molecules containing $\mathrm{B}-\mathrm{C}$ bonds. ${ }^{4}$ The properties of the $\mathrm{B}-\mathrm{B}$ bond of these molecules is the key factor in the synthesis reactions. While being thermodynamically stable, ${ }^{5}$ its Lewis acid character favors its activation upon interaction with bases. ${ }^{4,6}$ The resulting adducts are precursors of the B-B bond cleavage for the formation of boron compounds. $^{7-11}$ Besides its employment in synthesis processes, due to this strong affinity with bases, diboron(4) compounds may be of potential interest as building blocks in the $2 \mathrm{D}$ supramolecular assembly on surfaces as well. The introduction

\footnotetext{
${ }^{a}$ Department of Chemical and Pharmaceutical Sciences, University of Trieste, 34127 Trieste, Italy.E-mail: fronzoni@units.it, acossaro@units.it

${ }^{b}$ IOM-CNR, Istituto Officina dei Materiali-CNR, S.S.14, Km 163.5, 34149 Trieste, Italy

${ }^{c}$ ISM-CNR, Istituto di Struttura della Materia, LD2 Unit, 34149 Trieste, Italy

${ }^{d}$ Elettra-Sincrotrone Trieste, 34149 Basovizza, Trieste, Italy

$\dagger$ Electronic supplementary information (ESI) available. See DOI: 10.1039/d1cp03428f
}

of functionalities on surfaces allows tailoring of intermolecular recognition processes and design of specific interaction schemes. ${ }^{12-14}$ To our knowledge, and quite surprisingly, the use of diboron molecules to this aim and, in general, the study of the chemistry of the B-B bond on surfaces is mostly unexplored yet. We have recently reported on the on-surface synthesis of a $2 \mathrm{D}$ covalent framework supported by the $\mathrm{Au}(111)$ surface, formed by a network of B-B bonded boroxine groups, ${ }^{15}$ which exhibits delocalized electronic states in the valence band, likely due to hybridization of the outer electronic states of the framework with the substrate. A proper description of the electronic properties of diboron systems on surfaces is mandatory for both exploring their use in synthesis processes catalyzed by the surface and describing the functionalities of $2 \mathrm{D}$ architectures they may be part of bis(catecholato)diboron (2-(1,3,2benzodioxaborol-2-yl)-1,3,2-benzodioxaborole, $\mathrm{B}_{2} \mathrm{Cat}_{2}$ in the following) (see Fig. 1) is, together with bis(pinacolato)diboron, among the most frequently adopted diboron precursors in the synthesis of boronic compounds. It is a relatively simple molecule we selected as a prototype of diboron species and we present here a combined theoretical and experimental study of its electronic properties by comparing photoemission (XPS) and near edge X-ray absorption spectroscopy (NEXAFS) with DFT calculations. Experiments have been performed both for the isolated molecule in the gas phase and on a poorly reactive surface like $\mathrm{Au}(111)$ to evidence dichroic effects in the 

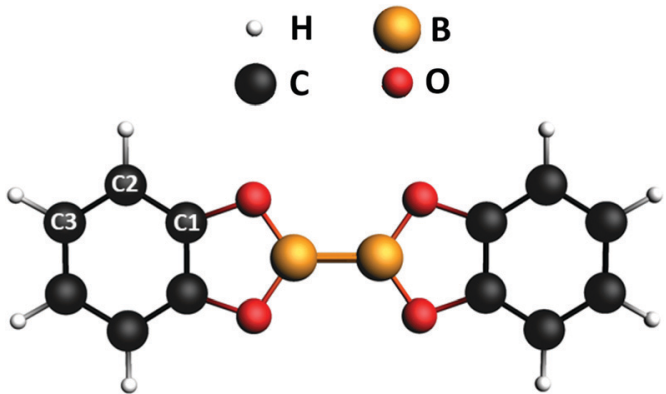

Fig. 1 Chemical structure of $\mathrm{B}_{2} \mathrm{Cat}_{2}$. The labels indicate the nonequivalent carbon atoms. The molecular plane corresponds to the $x y$ plane, with the direction of the $\mathrm{B}-\mathrm{B}$ bond along the $\mathrm{x}$ axis.

absorption. Dichroic effects on the $\mathrm{Au}(111)$ surface have been studied by measuring NEXAFS spectra in p-polarization (p-pol), i.e. with the light electric field plane perpendicular to the metal surface, and in s-polarization (s-pol), i.e. with the light electric field plane parallel to the metal surface.

From a general perspective, the theoretical description of $\mathrm{B}$ 1s core excitations of boronic- and B-B containing systems is a challenging task, as static correlation effects between different excited states need to be taken into account. ${ }^{16}$ This can be done using wave function based approaches, which becomes however prohibitively expensive when the size of the system increases, as in the case of the $\mathrm{B}_{2} \mathrm{Cat}_{2}$ molecule. Therefore, it is necessary to search for an alternative, less expensive method to calculate the B 1 s core excited states. In the $\triangle \mathrm{SCF}-\mathrm{DFT}(\triangle \mathrm{SCF})$ method, based on the energy difference between two selfconsistent-field (SCF) calculations, the core excited state is found by promoting an electron from the core orbital to one of the unoccupied orbitals in the ground state. The MOs are then re-optimized for each excited electron configuration and a different set of MOs for each excited state is obtained. Relaxation changes among different excited-state configurations relative to the same core hole are therefore taken into account at the $\triangle \mathrm{SCF}$ level, while they are not considered at the DFT level employing transition potential (TP) approximation ${ }^{17,18}$ (DFT-TP), which employs the same fixed set of orbitals obtained from a single TP calculation to describe the entire excited state manifold.

We show here how the $\triangle$ SCF calculations better reproduce the B K-edge NEXAFS experimental spectrum with respect to both DFT-TP and time-dependent DFT (TDDFT). We give a detailed description of the transitions corresponding to the peaks of the NEXAFS O, B and C K-edges and to the geometry of the related molecular orbitals.

\section{Theoretical methods and computational details}

The ground-state equilibrium geometry of $\mathrm{B}_{2} \mathrm{Cat}_{2}$ was computed at the density functional theory (DFT) ${ }^{19}$ level, employing the hybrid B3LYP xc functional and a basis set of Slater-type orbitals (STO) of triple-zeta quality (TZP) for all atoms. Calculations were performed by using the Amsterdam Density Functional (ADF) quantum chemistry code. ${ }^{20}$ The geometrical parameters (bond lengths and bond angles) of the optimized geometry are presented in Fig. S1 and Table S1 of the ESI $\dagger$ and show a close agreement with the experimental data derived from the crystallographic structure. ${ }^{21}$ The molecule is planar $\left(D_{2 \mathrm{~h}}\right.$ point group symmetry) and the molecular plane corresponds to the $x y$ plane in the molecular frame, with the B-B bond along the $x$ direction (see Fig. 1).

The XPS and NEXAFS spectra at the B 1s, C 1s and O 1s edges were computed at the DFT level employing the PW86xPerdew (PW86) generalized gradient approximation (GGA) for the xc functional $^{22,23}$ as implemented in the ADF program..$^{20,24}$

In order to accurately describe higher excitations close to the ionization threshold, an even tempered Quadruple-Zeta basis set with three polarization and three diffuse functions (designated as ET-QZ3P-3DIFFUSE in the ADF database) has been employed for the core-excited atom (B, C or $\mathrm{O}$, respectively), while a TZP (Triple Zeta + polarization) basis set was used for the remaining atoms. In particular, a frozen core TZP.1s basis set has been employed for the $\mathrm{B}, \mathrm{C}$ and $\mathrm{O}$ non-excited atoms, to ensure the correct localization of the half core hole.

For the XP spectrum simulation, the B 1s, O 1s and C 1s core Ionization Potentials (IPs) have been calculated at the $\triangle \mathrm{SCF}$ Kohn-Sham level allowing a full relaxation of the ionized core hole. The energy of each $1 \mathrm{~s}^{-1}$ ionic state has been obtained through a SCF unrestricted calculation. The relative intensity among different primary lines is simply assumed to be proportional to the number of equivalent atoms of the same type. This is a good approximation since the XPS spectra were measured with a photon energy well above the considered ionization thresholds.

The $\mathrm{B}, \mathrm{O}$ and $\mathrm{C}$ K-edge NEXAFS spectra at each nonequivalent atomic center have been computed with the DFTTP method in which half an electron is removed from the initial core orbital and all the orbitals are relaxed until self-consistency is obtained. Relaxation effects upon the core-hole formation are usually adequately included in the DFT-TP approach. ${ }^{25}$ The performance of the hybrid B3LYP potential ${ }^{26-28}$ has also been tested considering the B 1s core excitation spectrum (see Fig. S5 of the ESI $\dagger$ ). No significant differences emerged from the comparison between the PW86 and B3LYP calculated spectra.

For each of the three non-equivalent $\mathrm{C}$ sites of the $\mathrm{B}_{2} \mathrm{Cat}_{2}$ molecule (see Fig. 1), a separate computation of the $\mathrm{C} 1 \mathrm{~s}$ excitation spectrum has been performed and the total spectrum has been obtained by summing up the partial contributions weighted for the number of equivalent atoms.

Within the DFT-TP scheme, the excitation energies correspond to the differences between the eigenvalues of the virtual orbitals and the 1s core orbitals referred to the TP configuration:

$$
\Delta E_{i \rightarrow f}=\varepsilon_{f}^{\mathrm{TP}}-\varepsilon_{i}^{\mathrm{TP}},
$$

while the transition intensities are expressed as oscillator strengths $f_{i \rightarrow f}$. For a free molecule $f_{i \rightarrow f}$ corresponds to

$$
f_{i \rightarrow f}=\frac{2}{3} n_{i} \Delta E_{i \rightarrow f}\left|\left\langle\varphi_{f}^{\mathrm{TP}}|\boldsymbol{\mu}| \varphi_{i}^{\mathrm{TP}}\right\rangle\right|^{2}
$$


and involves matrix elements of the electric dipole operator between initial and final TP MOs, where $n_{i}$ denotes the occupation number of the core orbital in the ground state. Since the DFT-TP approach leads generally to a less attractive potential and consequently the excitation energies are generally too large, they have been shifted with respect to the $\Delta$ IP value by an amount given by the energy difference $\varepsilon_{1 \mathrm{~s}}^{\mathrm{TP}}-\Delta \mathrm{SCF}(1 \mathrm{~s})$. The energy of the $\triangle \mathrm{SCF} 1 \mathrm{~s}^{-1}$ ionic state corresponds to that previously calculated for the XP spectra.

Due to the discrepancies between experiments and the theoretical DFT-TP results of the B 1s NEXAFS spectrum, the $B$ 1s core excitations of the gas-phase $B_{2} \mathrm{Cat}_{2}$ have been also computed at the TDDFT level, which formally includes the coupling between the single excited configurations, employing different xc functionals with the ground state electron configuration. The TDDFT computational details and the results are reported in Section $\mathrm{S} 4$ of the ESI, $\dagger$ which show that no improvement is obtained at the TDDFT level compared to the simpler DFT-TP scheme.

To better describe electronic relaxation effects, the B 1s core excitations and oscillator strengths have been also calculated at the $\triangle \mathrm{SCF}$ level employing the procedure described in the following. In the $\triangle$ SCF method, the initial $\left(\Psi_{i}\right)$ and final $\left(\Psi_{f}\right) \mathrm{N}-$ electron wave functions entering the dipole matrix element (computed in the length gauge of the dipole operator),

$$
\mu_{i \rightarrow f}=\left\langle\Psi_{f}|\hat{\mu}| \Psi_{i}\right\rangle,
$$

are Slater determinants constructed from Kohn-Sham molecular orbitals (MOs) obtained with the SCF procedure relative to the ground state (GS) and excited state occupation numbers, respectively. The GS MOs are obtained from a spin-restricted calculation, while the excited-state MOs are calculated within a spin polarized scheme with $N_{\alpha}-N_{\beta}=0$, where $N_{\alpha}$ and $N_{\beta}$ denote the numbers of spin-up and spin-down electrons, respectively. In the specification of the occupation numbers, we removed a $\beta$ electron from the B 1s core. Singlet coreexcitation energies are obtained according to the spinpurification formula. ${ }^{29}$ Since the two sets of MOs, $\left\{\varphi_{\mu}^{i}\right\}$ and $\left\{\varphi_{\lambda}^{f}\right\}$, used to construct $\Psi_{i}$ and $\Psi_{f}$, respectively, are nonorthogonal, the $N$-electron matrix elements in eqn (3) are evaluated with the general rules for non-orthogonal spin orbitals derived by Löwdin. ${ }^{30}$ Denoting with $S_{f i}$ the overlap matrix between the two sets of occupied MOs, $\left(S_{f i}\right)_{\lambda \mu}=\left\langle\varphi_{\lambda}^{f} \mid \varphi_{\mu}^{i}\right\rangle, \mu_{i \rightarrow f}$ of eqn (3) can be written as

$$
\left\langle\Psi_{f}|\hat{\mu}| \Psi_{i}\right\rangle=\sum_{\lambda \mu}\left\langle\varphi_{\lambda}^{f}|\hat{\mu}| \varphi_{\mu}^{i}\right\rangle \operatorname{adj}\left(S_{f i}\right)_{\lambda \mu},
$$

in terms of dipole matrix elements between the two sets of MOs and the adjugate of $S_{f i}$ (i.e. the transpose of its cofactor matrix). When $\left\langle\Psi_{f} \mid \Psi_{i}\right\rangle=\operatorname{det}\left(S_{f i}\right) \neq 0$, eqn (4) reduces to

$$
\left\langle\Psi_{f}|\hat{\mu}| \Psi_{i}\right\rangle=\operatorname{det}\left(S_{f i}\right) \sum_{\lambda \mu}\left\langle\varphi_{\lambda}^{f}|\hat{\mu}| \varphi_{\mu}^{i}\right\rangle\left(S_{f i}^{-1}\right)_{\lambda \mu} .
$$

The origin independence of the transition matrix elements is enforced by adding the dipole of the nuclear charges, weighted by the overlap $\left\langle\Psi_{f} \mid \Psi_{\mathrm{i}}\right\rangle$. Results of a recent study ${ }^{31}$ indicate that this correction gives results of accuracy comparable to that obtained by enforcing exact orthogonality of $\Psi_{i}$ and $\Psi_{f}$. In any case, when exact orthogonality is not enforced by symmetry, we checked that the overlap between the initial and final states is rather small ( $\sim 10^{-3}$ or smaller).

In order to compare the theoretical results with the NEXAFS experimental measurements recorded for the $\mathrm{B}_{2} \mathrm{Cat}_{2}$ molecule adsorbed on the $\mathrm{Au}(111)$ surface (in s- and p-polarization), we have considered the molecule fixed in space and have calculated the NEXAFS p- and s-pol intensities employing the following formula for the oscillator strength:

$$
f_{i \rightarrow f}=2 \Delta E_{i \rightarrow f}\left|\left\langle\Psi_{f}|\bar{e} \cdot \bar{\mu}| \Psi_{i}\right\rangle\right|^{2}
$$

where $\Psi_{i}$ and $\Psi_{f}$ are the initial and final TP MOs in the DFT-TP calculations and Slater determinants with the GS and excited state occupation numbers, respectively, in the $\triangle \mathrm{SCF}$ calculations.

The $\triangle \mathrm{SCF} B \mathrm{~s}, \mathrm{C} 1 \mathrm{~s}$ and $\mathrm{O} 1 \mathrm{~s}$ IPs are presented in the figure of the corresponding NEXAFS spectrum and mark a separation of the whole spectral region into two sub-regions with different physical meanings. Only transitions that occur below the ionization threshold IP can be accurately described by the employed computational protocol. Above the IP only qualitative information can be extracted since the electronic continuum wave function cannot be properly described with standard basis sets of quantum chemistry programs.

\section{Results and discussion}

In the first part of the discussion the experimental XPS and NEXAFS spectra at the $\mathrm{B} 1 \mathrm{~s}, \mathrm{O}$ 1s and $\mathrm{C} 1 \mathrm{~s}$ edges of the gas phase $\mathrm{B}_{2} \mathrm{Cat}_{2}$ will be compared with the theoretical results in order to achieve an assignment of the spectral features. In the second part, the polarization dependent NEXAFS measurements of the $\mathrm{B}_{2} \mathrm{Cat}_{2}$ adsorbed on the $\mathrm{Au}(111)$ surface will be analysed with the support of the theoretical results obtained for the fixed-in-space molecule. The experimental data have been acquired at the Gas Phase beamline ${ }^{32}$ and at the ANCHORSUNDYN endstation ${ }^{33}$ at the Elettra Synchrotron. Experimental details are reported in the ESI. $\dagger$

\section{Gas phase results}

$B$ 1s, $C$ 1s and $O$ 1s photoelectron spectra. The experimental XPS spectra of the $\mathrm{B} 1 \mathrm{~s}, \mathrm{C} 1 \mathrm{~s}$ and $\mathrm{O} 1 \mathrm{~s}$ core levels of $\mathrm{B}_{2} \mathrm{Cat}_{2}$ together with the calculated $\triangle \mathrm{SCF}$ ionization potentials (IPs) for the $\mathrm{B}, \mathrm{C}$ and $\mathrm{O}$ atoms are reported in the ESI $\dagger$ (Fig. S2, S3 and $\mathrm{S} 4$, respectively). Here we briefly comment on the main features observed. The experimental XP spectrum of the B 1s core level (Fig. S2, ESI $\dagger$ ) shows a sharp intense peak at $196.89 \mathrm{eV}$ which is close to the main B 1s peak at $197.53 \mathrm{eV}$ of gas-phase catecholborane. ${ }^{34}$ The latter is the molecule whose structure corresponds to half $\mathrm{B}_{2} \mathrm{Cat}_{2}$, with a terminal $\mathrm{H}$ bonded to the $\mathrm{B}$ atom. The difference in the $\mathrm{B} 1 \mathrm{~s}$ binding energy we reported has therefore to be ascribed to the different chemical environment given by the presence of the B-B bond. The two much weaker structures here observed at 202.13 and $202.71 \mathrm{eV}$ are attributed 
to satellite states of the B 1s core level like those at 200.77 and $203.25 \mathrm{eV}$ of catecholborane. The many-body nature of these structures cannot be described by the present computational approach.

The $\mathrm{C}$ 1s photoelectron spectrum of $\mathrm{B}_{2} \mathrm{Cat}_{2}$ is presented in Fig. S3 of the ESI $\dagger$ together with the calculated $\Delta$ SCF IPs of the three nonequivalent carbon atoms of the molecule (C1, C2 and C3, see Fig. 1). The two experimental peaks are assigned to the C2, C3 sites (first peak at $290.49 \mathrm{eV}$ ) and to the C1 site (second peak, at $291.94 \mathrm{eV})$; their energy splitting $(1.45 \mathrm{eV})$ is a consequence of the reduced shielding of the $\mathrm{C} 1$ site, which is adjacent to the $\mathrm{O}$ atom, with respect to $\mathrm{C} 2$ and $\mathrm{C} 3$ sites. Calculations provide a chemical shift of $131 \mathrm{meV}$ between the C2 and C3 sites with the lower binding energy associated to C3. The experimental line shape shows a pronounced asymmetry, detected also in catecholborane ${ }^{34}$ and attributed to vibrational effects. The spectrum was fitted with a function of three skew gaussians and the extracted peak positions are 291.95, 290.58 and $290.38 \mathrm{eV}$ in accordance with the calculated IPs.

The $\mathrm{O}$ 1s photoelectron spectrum of $\mathrm{B}_{2} \mathrm{Cat}_{2}$ is shown in Fig. S4 of the ESI. $\dagger$ The peak at $539.03 \mathrm{eV}$ represents the IP of the four equivalent oxygen atoms, as also indicated by the calculated $\triangle$ SCF IP line. The second intense peak around $541 \mathrm{eV}$ is due to the $\mathrm{O} 1 \mathrm{~s}$ signal of $\mathrm{CO}_{2}$ that we dosed together with $\mathrm{B}_{2} \mathrm{Cat}_{2}$ in order to have a known feature in the spectra to properly calibrate the photon energy scale. Details about the calibration references we used are reported in the ESI. $\dagger$

B 1s, C 1s and $O$ 1s NEXAFS spectra. The B K-edge NEXAFS experimental spectrum is shown in Fig. 2 together with the theoretical results relative to the $\triangle \mathrm{SCF}$ and DFT-TP calculations. An intense lower energy peak (peak A at $191.362 \mathrm{eV}$ ) dominates the experimental spectrum, followed by a weaker double-peaked feature between 194 and $195 \mathrm{eV}$ and by a poorly structured signal towards a higher energy. The DFT-TP results (lower panel of Fig. 2) show significant discrepancies with the experiment; in particular, the calculations do not reproduce correctly the intensity distribution between the first peak and the feature around $195 \mathrm{eV}$ for which the calculations overestimate the oscillator strengths for the transitions involved. The inability of TDDFT to correctly describe the $\mathrm{B}$ 1s core excitations emerges in the case of the $\mathrm{B}_{2} \mathrm{Cat}_{2}$ molecule, as shown by the results presented in Fig. S6 of the ESI: $\dagger$ the discrepancies with respect to the $\mathrm{B} 1 \mathrm{~s}$ experimental spectrum persist in terms of both energy separation and intensity distribution irrespective of the exchange-correlation functional employed (LB94, B3LYP and CAM-B3LYP).

The origin of the incorrect intensity distribution calculated at the DFT-TP level for the B 1s transitions has been already discussed in previous studies on boronic acid derivatives. ${ }^{16,35}$ We demonstrated that to correctly reproduce the B 1s NEXAFS spectrum one needs to include static correlation effects beyond those described by the coupling of singly-excited configurations, as in the TDDFT approach. In ref. 16 this was achieved by performing $a b$ initio Multi-Configuration Self-Consistent-Field (MCSCF) calculations. We are led to assume that the inclusion of residual static correlation effects plays an important role in

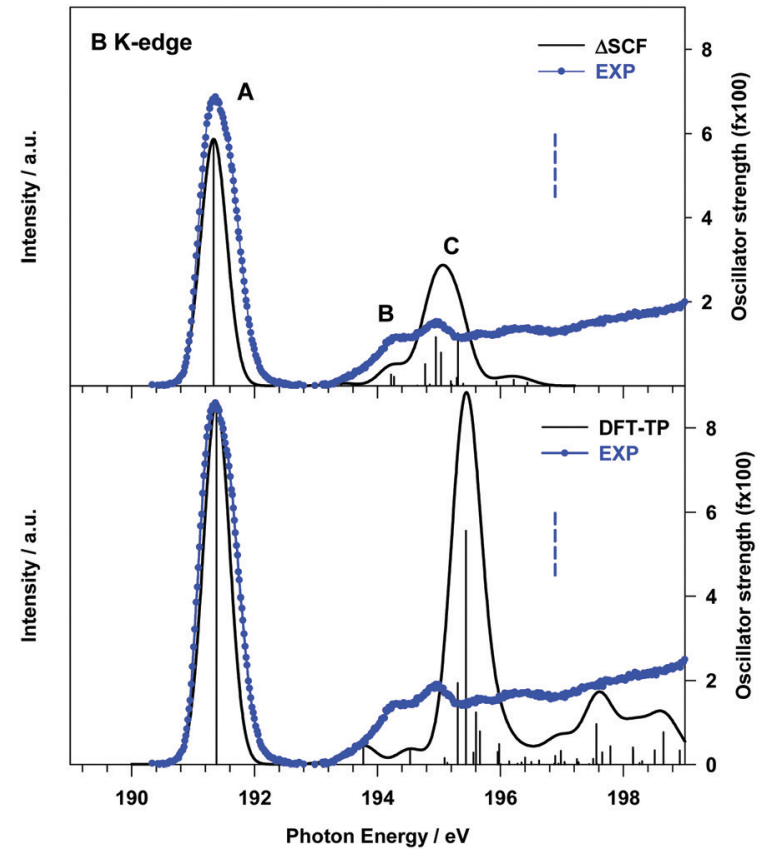

Fig. 2 B K-edge gas phase NEXAFS spectrum of $\mathrm{B}_{2} \mathrm{Cat}_{2}$. Upper panel: Comparison with the theoretical $\triangle \mathrm{SCF}$ results; lower panel: comparison with the theoretical DFT-TP results. The theoretical spectra have been shifted by $+0.45 \mathrm{eV}$ ( $\Delta \mathrm{SCF}$ spectrum) and $+0.17 \mathrm{eV}$ (DFT-TP spectrum) in order to match the first experimental peak. The stick spectra are broadened by using a Gaussian line shape with FWHM $=0.5 \mathrm{eV}$. The experimental B 1s IP (196.89 eV) is indicated with a vertical dashed line.

the description of the $\mathrm{B} 1 \mathrm{~s}$ photoexcitation of $\mathrm{B}_{2} \mathrm{Cat}_{2}$, as also evidenced by the shake-up structures of the XPS B 1s spectrum in Fig. S2 (ESI; † see the "B 1s, C 1s and O 1s photoelectron spectra" section).

The effect of a more accurate treatment of relaxation, which is the dominant electron correlation effect at play in core excitation processes, is apparent from the comparison between the B 1s experimental NEXAFS spectrum and the $\triangle \mathrm{SCF}$ results, presented in the upper panel of Fig. 2.

A significant improvement of the description of the experimental spectrum is obtained compared to DFT-TP results, in particular as concerns the intensity distribution among the spectral features. The $\triangle \mathrm{SCF}$ spectrum has been obtained by calculating, for each dipole-allowed final symmetry, the first six excitation energies and transition dipole moments. This choice has been supported by the analysis of the DFT-TP results, which allows the identification of the final MOs involved in the description of the main spectral features below the edge. The $\triangle$ SCF B $1 \mathrm{~s}$ core excitation energies and oscillator strengths are presented in Table 1, together with the attribution of the spectral features and the experimental data. The first calculated peak A arises from the $B$ 1s transition to the LUMO orbital $\left(7 b_{1}\right)$ which has a predominant $\pi(\mathrm{B}-\mathrm{B})$ bonding character (out of plane (oop) B 2p atomic components) with minor $\pi^{*}(\mathrm{~B}-\mathrm{O})$ antibonding contributions (the 3D plot of the $7 \mathrm{~b}_{1}$ final MO is presented in Fig. S7 of the ESI $\dagger$ ).

The two main transitions of feature C (at 194.95 and $195.31 \mathrm{eV}$ ) involve final MOs with mainly valence $\pi^{*}(\mathrm{~B}-\mathrm{B})$ (out of plane) 
Table 1 B 1s $\triangle$ SCF excitation energies (eV) and oscillator strengths $f$ of the main transitions of $\mathrm{B}_{2} \mathrm{Cat}_{2}$

\begin{tabular}{|c|c|c|c|c|}
\hline Peak & $\begin{array}{l}\Delta \mathrm{SCF} \\
\text { transition }^{a}\end{array}$ & $E^{b}(\mathrm{eV})$ & $f \times 100^{c}$ & Assignment, main character of the final MO \\
\hline A & B $1 \mathrm{~s} \rightarrow 7 \mathrm{~b}_{1}$ & 191.33 & 5.87 & $\pi_{\text {out of plane }}(\mathrm{B}-\mathrm{B})+\pi^{*}(\mathrm{~B}-\mathrm{O})$ \\
\hline \multirow[t]{2}{*}{ B } & B $1 \mathrm{~s} \rightarrow 29 \mathrm{a}_{1}$ & 194.22 & 0.27 & Rydberg (in plane AO components) \\
\hline & B $1 \mathrm{~s} \rightarrow 9 \mathrm{~b}_{1}$ & 194.27 & 0.21 & $\pi^{*}(\mathrm{C}-\mathrm{C})$ \\
\hline \multirow[t]{4}{*}{$\mathrm{C}$} & B $1 \mathrm{~s} \rightarrow 23 \mathrm{~b}_{2}$ & 194.78 & 0.52 & Mixed valence $\pi_{\text {in plane }}(\mathrm{B}-\mathrm{B}) /$ Rydberg \\
\hline & B $1 \mathrm{~s} \rightarrow 10 \mathrm{~b}_{1}$ & 194.95 & 1.15 & Mixed valence $\pi_{\text {out of plane }}^{*}(\mathrm{~B}-\mathrm{B}) /$ Rydberg \\
\hline & B $1 \mathrm{~s} \rightarrow 24 \mathrm{~b}_{2}$ & 195.03 & 0.79 & Mixed valence $\pi_{\text {in plane }}(\mathrm{B}-\mathrm{B}) /$ Rydberg \\
\hline & B $1 \mathrm{~s} \rightarrow 11 \mathrm{~b}_{1}$ & 195.31 & 1.25 & Mixed valence $\pi_{\text {out of plane }}^{*}(\mathrm{~B}-\mathrm{B}) /$ Rydberg \\
\hline
\end{tabular}

${ }^{a}$ When the core hole is localized on the B site the molecular symmetry lowers from $D_{2 \mathrm{~h}}$ to $C_{2 \mathrm{v}}$ and the molecular plane is $y z$ with the B-B bond along the $z$ axis. ${ }^{b}$ Calculated excitation energies shifted by $0.45 \mathrm{eV}$ to match the first experimental peak. ${ }^{c}$ Only calculated transitions with $f \times 100$ $\geq 0.20$ are reported.

antibonding character mixed with diffuse atomic components, while MOs with $\pi(\mathrm{B}-\mathrm{B})$ (in plane) bonding character, mixed with diffuse components, characterize the other two transitions at 194.78 and $195.03 \mathrm{eV}$ (see Fig. S7 of the ESI $\dagger$ ). The lower energy tail of C, labelled B in Fig. 2, arises from two low intensity transitions, one of diffuse nature (at $194.22 \mathrm{eV}$ ) and the other of mainly $\pi^{*}(\mathrm{C}=\mathrm{C})$ antibonding character (at $194.27 \mathrm{eV}$ ).

The analysis of the contributions to the transition dipole moment (see eqn (4) and (5)) for the $\Delta$ SCF $9 a_{1}\left(B 1 s^{-1}\right) \rightarrow 7 b_{1}$ transition reveals that the intensity is mainly contributed by the $\left\langle\varphi_{7 \mathrm{~b}_{1}}^{f}|\hat{\mu}| \varphi_{9 \mathrm{a}_{1}}^{i}\right\rangle$ dipole matrix element between the initial 9a $\mathrm{a}_{1} \mathrm{MO}$ of the GS and the $7 b_{1}$ MO of the $\triangle$ SCF excited state. The value of the cofactor, which multiplies the dipole integral, is close to 1 (0.918), and thus it does not influence the calculated intensity of this transition. On this ground, the relatively large oscillator strength computed for the $\triangle \mathrm{SCF} 9 \mathrm{a}_{1} \rightarrow 7 \mathrm{~b}_{1}$ transition can be related to the composition of the $7 b_{1}$ final $\mathrm{MO}$, notably the significant participation of the $\mathrm{B} 2 \mathrm{p}$ atomic components, which carries most of the dipole transition moment. Considering the transitions of peak $\mathrm{C}$, this kind of analysis still points out that the intensity of each transition is dominated by the contribution of the dipole matrix element between the initial $9 \mathrm{a}_{1} \mathrm{MO}$ (B $1 \mathrm{~s}^{-1}$ ) of the GS and the final MO of the $\triangle \mathrm{SCF}$ transition considered.

Also the relative intensity of these transitions, compared to the first peak, can be correlated to the atomic composition of the final $\mathrm{MO}$, in particular the $\mathrm{B} 2 \mathrm{p} / \mathrm{np}$ atomic contributions, which turn out to be reduced with respect to the final $7 b_{1}$ MO of the first transition.

The significant decrease of the oscillator strength obtained at the $\triangle \mathrm{SCF}$ level compared to the DFT-TP ones for the transitions of peak $\mathrm{C}$ can be mainly ascribed to the decreased value of the dipole integral of the corresponding transitions and therefore to the relaxation changes among different excited state configurations neglected in the DFT-TP approach, which treats the relaxation effects in an average way. We underline that the value of the cofactor multiplying the dominant dipole integral of the transitions of peak $\mathrm{C}$ is lower (around 0.8) than the one corresponding to the $9 \mathrm{a}_{1}\left(\mathrm{~B} 1 \mathrm{~s}^{-1}\right) \rightarrow 7 \mathrm{~b}_{1}$ transition and the calculated intensity is further reduced compared to the DFT-TP results.

The intensity distribution as well as the energy separation between the $\pi(\mathrm{B}-\mathrm{B})$ bonding and the $\pi^{*}(\mathrm{~B}-\mathrm{B})$ anti-bonding final states we described here represents a sensitive monitor of the chemistry the molecule is involved in, recalling what we discussed in the introduction. The Lewis acid character of the molecule is expected to be related to these orbitals, as they are localized on the $\mathrm{B}$ atoms.

The gas phase experimental C K-edge NEXAFS spectrum of $\mathrm{B}_{2} \mathrm{Cat}_{2}$ is shown in Fig. 3 together with the theoretical DFT-TP results. The calculated excitation energies and oscillator strengths are presented in Table 2, along with their assignment to single-particle transitions. Two main experimental peaks characterize the low energy region (around 285 and $287 \mathrm{eV}$, respectively), while several less intense and broader structures are present in going towards the ionization threshold. The spectrum resembles the one measured for catecholborane, ${ }^{34}$ as expected due to the same chemical configuration for the carbon atoms in the two cases.

The comparison with the theory points out a good agreement for the groups of excited states which contribute to the first structures (A, B and C, with C describing the higher energy tail of the second experimental band). Conversely, a poorer agreement is observed at a higher energy, in particular in the energy region around $290 \mathrm{eV}$ with an overestimation of the intensity provided by the calculations. Indeed, the close

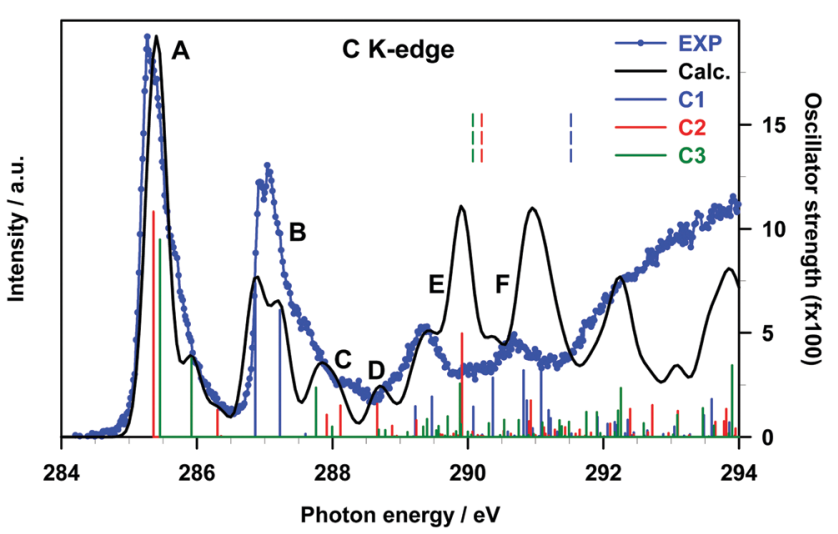

Fig. $3 \mathrm{C}$ K-edge gas phase NEXAFS spectra of $\mathrm{B}_{2} \mathrm{Cat}_{2}$. The DFT-TP theoretical spectrum has been rigidly shifted by $-0.37 \mathrm{eV}$ on the experimental energy scale. The main calculated structures are labelled with capital letters. Colored solid lines represent the partial $\mathrm{Ci}$ contributions. $\triangle$ SCF C 1s IPs (C1: $291.89 \mathrm{eV}$; C2: $290.58 \mathrm{eV;} \mathrm{C3:} 290.45 \mathrm{eV}$ ) are indicated with vertical dashed lines. The stick spectrum is broadened by using a Gaussian line shape with FWHM $=0.35 \mathrm{eV}$. 
Table 2 DFT-TP C 1 s excitation energies (eV) and oscillator strengths $f$ of $\mathrm{B}_{2} \mathrm{Cat}_{2}$

\begin{tabular}{llrll}
\hline Peak & $E^{a}(\mathrm{eV})$ & \multicolumn{1}{c}{$f^{b}$} & Site & Assignment \\
\hline A & 285.36 & 10.81 & C2 & $\pi^{*}(\mathrm{C}-\mathrm{C})+\pi(\mathrm{B}-\mathrm{B})$ (LUMO) \\
& 285.46 & 9.49 & $\mathrm{C} 3$ & $\pi^{*}(\mathrm{C}-\mathrm{C})+\pi(\mathrm{B}-\mathrm{B})$ (LUMO) \\
& 285.92 & 3.79 & $\mathrm{C} 3$ & $\pi^{*}(\mathrm{C}-\mathrm{C})+\pi(\mathrm{B}-\mathrm{B})$ (LUMO+1) \\
$\mathrm{B}$ & 286.30 & 1.30 & $\mathrm{C} 2$ & \\
& 286.86 & 7.38 & $\mathrm{C} 1$ & $\pi^{*}(\mathrm{C}-\mathrm{C})+\pi(\mathrm{B}-\mathrm{B})$ (LUMO) \\
$\mathrm{C}$ & 287.22 & 6.08 & $\mathrm{C} 1$ & $\pi^{*}(\mathrm{C}-\mathrm{C})+\pi(\mathrm{B}-\mathrm{B})$ (LUMO+1) \\
& 287.76 & 2.38 & $\mathrm{C} 3$ & Rydberg (in plane components) \\
$\mathrm{D}$ & 288.12 & 1.51 & $\mathrm{C} 2$ & \\
$\mathrm{E}$ & 288.66 & 1.60 & $\mathrm{C} 2$ & \\
& 289.23 & 1.47 & $\mathrm{C} 1$ & \\
& 289.47 & 1.94 & $\mathrm{C} 1$ & \\
& 289.89 & 2.57 & $\mathrm{C} 3$ & Mixed $\pi^{*}(\mathrm{C}-\mathrm{C}) /$ Rydberg \\
& 289.91 & 4.95 & $\mathrm{C} 2$ & \\
& 290.08 & 1.45 & $\mathrm{C} 1$ & Rydberg (in plane components) \\
& 290.37 & 2.85 & $\mathrm{C} 1$ & Mixed $\pi(\mathrm{B}-\mathrm{B}) /$ Rydberg (in plane \\
$\mathrm{F}$ & 290.82 & 3.21 & $\mathrm{C} 1$ & components) \\
& 291.08 & 3.33 & $\mathrm{C} 1$ &
\end{tabular}

${ }^{a}$ Calculated excitation energies shifted by $-0.37 \mathrm{eV}$ to match the first experimental peak. ${ }^{b}$ Only the main transitions contributing to the theoretical features are presented.

proximity of the ionization region, above which the discretization of the non-resonant continuum affects the calculations, can explain the only qualitative description of the intensity distribution around and above $290 \mathrm{eV}$. Nonetheless, the attribution of the experimental features, which we will present in the following, can still be considered with confidence.

Band A arises from transitions from the $\mathrm{C} 2$ and $\mathrm{C} 3$ sites of the phenyl rings, namely the two $\mathrm{C} 1 \mathrm{~s} \rightarrow$ LUMO transitions, which contribute to the main peak, and the two $\mathrm{C} 1 \mathrm{~s} \rightarrow$ LUMO+1 transitions, which contribute to the higher energy tail of peak A (see Table 2). The same transitions from the $\mathrm{C} 1$ site, which is bonded to the $\mathrm{O}$ atom of the catechol moieties, are shifted to higher energies and give rise to peak $\mathrm{B}$. The energy separation between the LUMO transitions of peaks A and B (about $1.4 \mathrm{eV}$ ) reflects the shift of IPs between $\mathrm{C} 2 / \mathrm{C} 3$ and $\mathrm{C} 1$ sites $(1.37 \mathrm{eV})$, denoting a predominance of initial state effects on these excitation energies. Both LUMO and LUMO+1 final orbitals are localized on the phenyl ring and present a $\pi^{*}(\mathrm{C}-\mathrm{C})$ antibonding character with a minor contribution from the $\pi(\mathrm{B}-\mathrm{B})$ bond between the $\mathrm{B}$ atoms connecting the two catechol moieties (a plot of the LUMO orbital with the core hole localized on the $\mathrm{C} 2$ site is presented in Fig. S8 of the ESI $\dagger$ ).

The higher energy tail of peak B (denoted as C in Fig. 3) as well as feature $\mathrm{D}$ and the lower energy shoulder (around $289.5 \mathrm{eV}$ ) of peak $\mathrm{E}$ are contributed by several low intensity transitions towards final MOs of diffuse nature (see Table 2). In the energy region of $289.5-291 \mathrm{eV}$ the agreement between the theory and experiment deteriorates, as previously commented: the calculations provide two more intense peaks ( $\mathrm{E}$ and $\mathrm{F}$ ) around the $\mathrm{C}$ 1s thresholds, while two broad features, slightly shifted to lower energies, are present in the experiment. The high intensity of $\mathrm{E}$ and $\mathrm{F}$ theoretical peaks mainly arises from transitions towards final MOs with a $\pi^{*}(\mathrm{C}-\mathrm{C})$ antibonding character mixed with some diffuse components starting from all the $\mathrm{C}$ sites (peak E) and from the C1 site (peak F). The O

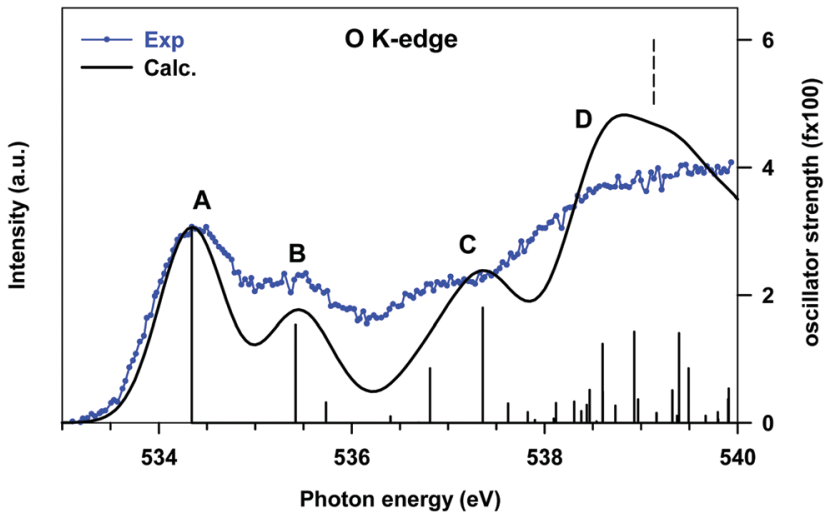

Fig. $4 \bigcirc \mathrm{K}$-edge gas phase NEXAFS spectra of $\mathrm{B}_{2} \mathrm{Cat}_{2}$. The DFT-TP theoretical spectrum has been shifted by $0.10 \mathrm{eV}$ in order to match the first experimental peak. The main calculated structures are labelled with capital letters. The $\triangle$ SCF O 1s IP (539.03 eV) is indicated with a vertical dashed line. The stick spectrum is broadened by using a Gaussian line shape with $\mathrm{FWHM}=0.8 \mathrm{eV}$.

Table 3 DFT-TP O 1s excitation energies (eV) and oscillator strengths $f$ of $\mathrm{B}_{2} \mathrm{Cat}_{2}$

\begin{tabular}{llll}
\hline Peak & $E^{a}(\mathrm{eV})$ & $f \times 100^{b}$ & Assignments \\
\hline A & 534.34 & 3.06 & $\pi_{\text {oop }}(\mathrm{B}-\mathrm{B})+\pi^{*}(\mathrm{~B}-\mathrm{O})$ \\
$\mathrm{B}$ & 535.42 & 1.54 & $\pi^{*}(\mathrm{C}=\mathrm{C})+\pi^{*}(\mathrm{C}=\mathrm{O})$ \\
C & 536.81 & 0.85 & Rydberg (in plane components $)$ \\
& 537.36 & 1.80 & \\
D & 538.60 & 1.24 & Mixed valence $\pi^{*}(\mathrm{~B}-\mathrm{B})+\pi^{*}(\mathrm{~B}-\mathrm{O}) /$ Rydberg \\
& 538.93 & 1.43 & Rydberg (in plane components $)$
\end{tabular}

${ }^{a}$ Calculated excitation energies shifted by $0.10 \mathrm{eV}$ to match the first experimental peak. ${ }^{b}$ Only the main transitions contributing to the theoretical features are reported.

K-edge NEXAFS experimental spectrum of gas phase $\mathrm{B}_{2} \mathrm{Cat}_{2}$ is compared with the DFT-TP theoretical spectrum in Fig. 4. The assignment of the main peaks in terms of the calculated excitation energies and oscillator strengths is presented in Table 3. The experimental spectrum shows two main features in the energy range up to $536 \mathrm{eV}$, followed by a quite smooth signal of increasing intensity towards the ionization threshold. The good agreement between the calculated and measured spectra confirms the adequacy of the DFT-TP scheme to describe the core excitations of the $\mathrm{O} 1 \mathrm{~s}$ channel in the $\mathrm{B}_{2} \mathrm{Cat}_{2}$ molecule.

The stronger calculated transition at $534.34 \mathrm{eV}$ gives rise to peak $A$ and involves the LUMO orbital which presents mainly a $\pi\left(\mathrm{B}-\mathrm{B}\right.$, out of plane) bonding character with a minor $\pi^{*}(\mathrm{~B}-\mathrm{O})$ antibonding contribution (see Fig. S9 of the ESI $\dagger$ ), similarly to the LUMO orbital obtained from the DFT-TP calculation with the core-hole localized on the B site.

Peak B mainly arises from the $\mathrm{O} 1 \mathrm{~s} \rightarrow \mathrm{LUMO}+1$ transition. The LUMO+1 MO is strongly localized on the phenyl ring $\left(\pi^{*}(\mathrm{C}=\mathrm{C})\right.$ character $)$ with only a weak conjugation with the $\mathrm{O}$ $2 \mathrm{p}_{z}$ components, consistent with the low intensity calculated for this transition. Features C and D are characterized by several low intensity transitions toward MOs of mainly diffuse nature. 
It is worth noting that the transition at $538.60 \mathrm{eV}$ (peak D) involves a final orbital which is the antibonding counterpart of the LUMO although several diffuse components are also present. The energy separation between the transitions towards the $\pi(\mathrm{B}-\mathrm{B})$ bonding and antibonding orbitals is about $4 \mathrm{eV}$, a value very close to that found for the corresponding transitions in the B 1s NEXAFS spectrum.

We underline the relatively low oscillator strengths provided by the DFT-TP calculations for the $\mathrm{O} 1 \mathrm{~s}$ transitions, which stem from the small $\mathrm{O} 2 \mathrm{p}$ atomic contribution to the virtual orbitals compared to the $\mathrm{C}$ and $\mathrm{B}$ atomic contributions. This is a sign of the polarization of the $\mathrm{C}-\mathrm{O}$ and $\mathrm{B}-\mathrm{O}$ bonds and of the large involvement of $\mathrm{O} 2 \mathrm{p}$ atomic components in the occupied MOs.

$\mathrm{B}_{2} \mathrm{Cat}_{2}$ on $\mathrm{Au}(111)$ NEXAFS results. The NEXAFS experimental spectra of $\mathrm{B}_{2} \mathrm{Cat}_{2}$ on $\mathrm{Au}(111)$ have been recorded at two different polarization angles of the electric field with respect to the surface. p-pol and s-pol geometries refer to the light polarization vector being normal and parallel to the surface plane, respectively. The calculated $\mathrm{p}$ - and s-pol spectra are relative to a gas-phase molecule oriented with the molecular plane fixed in the $x y$ plane of the molecular frame (see the Theoretical methods and computational details section). All B 1s, C 1s and O 1s spectra show a strong dichroism between s-pol and p-pol spectra, with the transitions to $\pi^{*}$ orbitals having a maximum intensity in p-pol. This indicates a flat adsorption geometry for the $\mathrm{B}_{2} \mathrm{Cat}_{2}$ on the $\mathrm{Au}(111)$ surface.

The B 1s experimental and calculated p- and s-pol spectra are presented in Fig. 5, in the energy region below the threshold. The intensity contribution from the p-pol component is prevalent in all the spectral region below the edge. The most remarkable dichroic effects are present in the region of the first experimental peak (around $191 \mathrm{eV}$ ) and are correctly reproduced by the calculations. This behavior reflects the $\pi$ symmetry of the final orbital $\left(7 b_{1}\right.$, LUMO) involved in the transition responsible for this peak. In the energy region above $194 \mathrm{eV}$

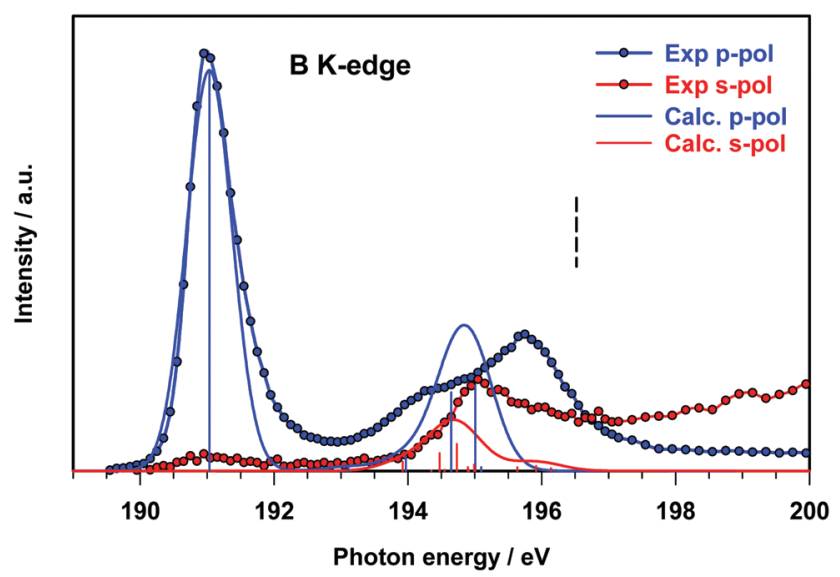

Fig. $5 \mathrm{~B}$ K-edge NEXAFS spectra of $\mathrm{B}_{2} \mathrm{Cat}_{2} \mathrm{QAu}(111)$ at two different polarization angles. The $\triangle S C F$ calculated spectra of the fixed in space $\mathrm{B}_{2} \mathrm{Cat}_{2}$ have been shifted by $0.15 \mathrm{eV}$ in order to match the first experimental peak. The $\triangle S C F B$ Is IP is indicated with a vertical dashed line. The stick spectra are broadened by using a Gaussian line shape with $\mathrm{FWHM}=0.80 \mathrm{eV}$. both p- and s-pol components are present. In particular, the spol component gives a significant contribution around $195 \mathrm{eV}$, which appears slightly underestimated by the theory and corresponds to the transitions encompassed by peak $\mathrm{C}$ in the gas phase spectrum, assigned to the excitations to the in-plane $\pi(\mathrm{B}-\mathrm{B}) \mathrm{MOs}$.

In this energy region the p-pol experimental component is still stronger than the s-pol one, with a large feature with a maximum around $196 \mathrm{eV}$, while the theory provides one p-pol peak mainly derived from the two out of plane $\pi^{*}(\mathrm{~B}-\mathrm{B})$ antibonding transitions.

Fig. 6 presents the $\mathrm{C}$ 1s experimental polarized spectra and their comparison with the calculated ones for the fixed in space $\mathrm{B}_{2} \mathrm{Cat}_{2}$ molecule. The p-pol component dominates in all the energy range below the threshold and the theory correctly describes the strong dichroic effects associated with the $\pi$ out of plane nature of most final MOs. In particular, the first two peaks correspond to the $\mathrm{A}$ and $\mathrm{B}$ peaks of the gas-phase spectrum (Fig. 3) associated to $\pi^{*}(\mathrm{C}-\mathrm{C})$ antibonding transitions.

Around $288 \mathrm{eV}$ the s-pol component starts to acquire some intensity with a feature corresponding to peak $\mathrm{C}$ of the gas phase spectrum due to the transitions towards diffuse MOs with in plane atomic components. Transitions of similar nature contribute to the experimental s-pol broad signal present in the energy range of 289-292 eV. The p-pol component contributes to this energy range with two broad features whose theoretical counterparts appear still overestimated and slightly shifted at a higher energy and correspond to the $\mathrm{E}$ and $\mathrm{F}$ peaks of the gas phase spectrum. This analysis of the $\mathrm{p}$ - and s-pol components of the C K-edge spectrum confirms the attribution of the spectral features proposed for the gas-phase experimental spectrum despite some discrepancies provided by the theory in the energy range around $\mathrm{C} 1 \mathrm{~s}$ thresholds.

The results for the $\mathrm{O}$ K-edge p-pol and s-pol spectra are shown in Fig. 7. The dichroic effects are still relevant at a lower

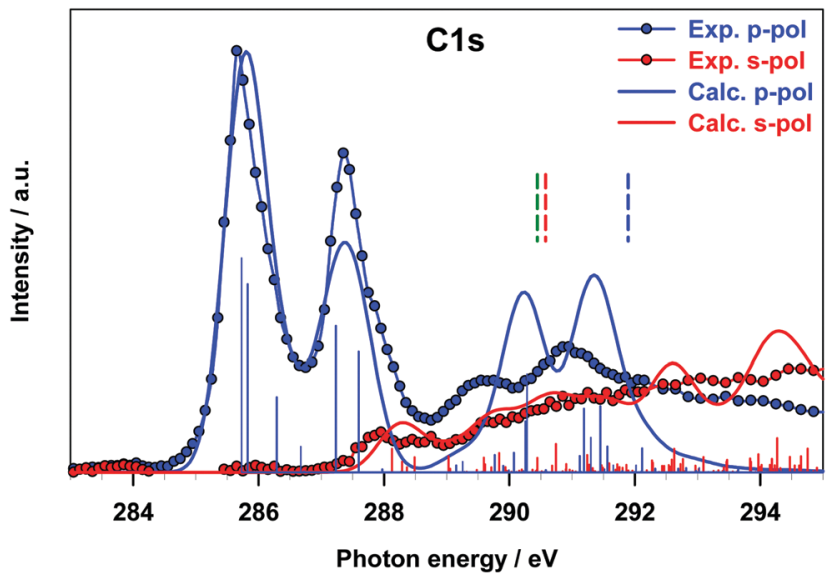

Fig. $6 \mathrm{C}$ K-edge NEXAFS spectra of $\mathrm{B}_{2} \mathrm{Cat}_{2} @ \mathrm{aAu}(111)$ at two different polarization angles. The DFT-TP calculated spectra of the fixed in space $\mathrm{B}_{2} \mathrm{Cat}_{2}$ have been reported without applying any energy shift. $\triangle \mathrm{SCF} C$ is IPs are indicated with vertical dashed lines. The stick spectra are broadened by using a Gaussian line shape with $\mathrm{FWHM}=0.75 \mathrm{eV}$. 


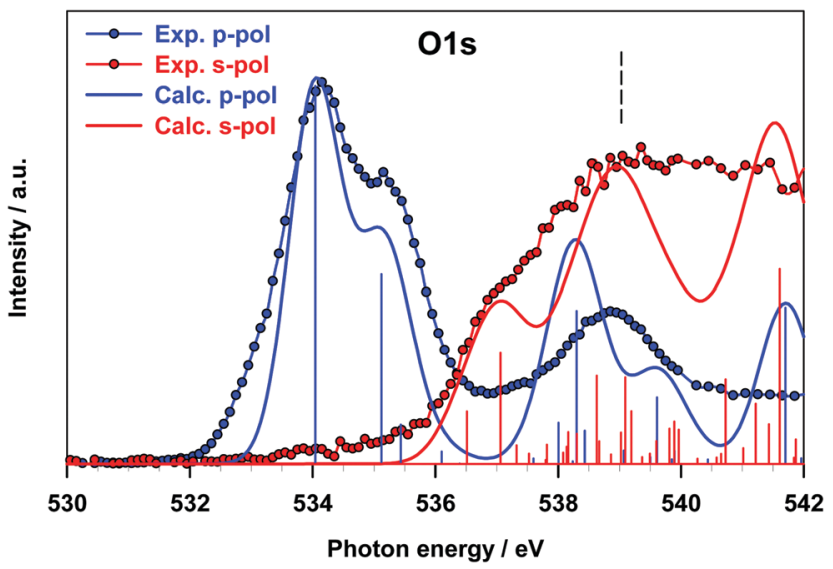

Fig. $7 \bigcirc \mathrm{K}$-edge NEXAFS spectra of $\mathrm{B}_{2} \mathrm{Cat}_{2} @ \mathrm{CAu}(111)$ at two different polarization angles. The DFT-TP calculated spectra of the fixed in space $\mathrm{B}_{2} \mathrm{Cat}_{2}$ have been shifted by $-0.20 \mathrm{eV}$ in order to match the first experimental peak. $\triangle$ SCF $O$ 1s IPs are indicated with vertical dashed lines. The stick spectra are broadened by using a Gaussian line shape with $F W H M=1.0 \mathrm{eV}$.

energy, while both components provide significant contributions to the features above $536 \mathrm{eV}$. The agreement between the experiment and theory is good for both components, and the strong dichroic effects present in the lower energy region are consistent with the $\pi$ nature of the transitions relative to the $\mathrm{A}$ and $\mathrm{B}$ peaks of the gas phase spectrum.

The intensity of the s-pol component mainly arises from transitions of diffuse nature (peak $\mathrm{C}$ of the gas phase spectrum), while the intensity distribution between the s- and p-pol components near the threshold reflects the character of the transitions contributing to peak D of the gas phase spectrum (see Table 3). The good agreement found between theoretical calculations on the oriented molecule and experimental results for $\mathrm{B}_{2} \mathrm{Cat}_{2}$ adsorbed on $\mathrm{Au}(111)$ proves that the $\mathrm{Au}$ surface does not perturb the transitions of any of the $\mathrm{B} 1 \mathrm{~s}, \mathrm{C} 1 \mathrm{~s}$ and $\mathrm{O} 1 \mathrm{~s}$ channels confirming the weak interaction of the molecule with the surface.

\section{Conclusions}

DFT calculations have been used to assign the features observed in the B, C and O K-shell XPS and NEXAFS spectra of the $\mathrm{B}_{2} \mathrm{Cat}_{2}$ molecule, with the aim of obtaining detailed information on the $\mathrm{B}_{2} \mathrm{Cat}_{2}$ electronic structure. The assignment of the absorption peaks observed in the gas-phase NEXAFS spectra has been validated by a study of the corresponding NEXAFS spectra for the molecule adsorbed on the poorly reactive $\mathrm{Au}(111)$ surface, which allowed confirmation of the nature of the virtual electronic states involved in the coreexcitation process. This represents a valuable reference to explore the surface chemistry of diboron compounds with other molecular species or with reactive metallic substrates. In particular the energy position and intensity of the B 1s core to the $\pi(\mathrm{B}-\mathrm{B})$ bonding and anti-bonding orbitals represent a sensitive probe of the chemistry of the molecule. In this regard, for example, we outline that, in the B 1s edge spectra, a quite different position of the first transition $(\sim 191 \mathrm{eV})$ is found with respect to the case of the covalent framework of boroxine groups based on the B-B bond $(\sim 194 \mathrm{eV}) \cdot{ }^{15}$ We suggest therefore that in the latter case a stronger interaction with the substrate was established and that the B-B centers were involved in a charge transfer process with a consequent change in their chemical environment.

Finally, our results point out that the $\triangle S C F$ method is required to describe the $\mathrm{B} 1 \mathrm{~s}$ core excited states in order to reach a good agreement with the experimental data, with respect to both DFT-TP and linear response TDDFT approaches. This indicates the importance of explicitly treating the relaxation changes among different excited-state configurations relative to the B 1s core hole, unlike the $\mathrm{C}$ and $\mathrm{O}$ K-edges which can be conveniently described at the DFT-TP level with the relaxation effects included in an average way. This behavior can be traced to the less pronounced relaxation following the formation of the shallower $\mathrm{B}$ 1s core hole compared to the heavier first row atoms, such as $\mathrm{C}$ and $\mathrm{O}$.

\section{Conflicts of interest}

There are no conflicts to declare.

\section{Acknowledgements}

The computational research was supported by Finanziamento per ricerca di ateneo, FRA 2018-2020 of the Università degli Studi di Trieste. The authors are grateful to the Stiftung Beneficentia for a generous grant employed to set up a computational server.

\section{Notes and references}

1 A. L. Korich and P. M. Iovine, Boroxine chemistry and applications: A perspective, Dalton Trans., 2010, 39, 1423-1431.

2 E. Dimitrijević and M. S. Taylor, Organoboron Acids and Their Derivatives as Catalysts for Organic Synthesis, ACS Catal., 2013, 3, 945-962.

3 G. Duret, R. Quinlan, P. Bisseret, N. Blanchard, A. R. Martin, J.-J. Vasseur and M. Smietana, Boron chemistry in a new light, Chem. Sci., 2013, 42, 5684-5713.

4 E. C. Neeve, S. J. Geier, I. A. I. Mkhalid, S. A. Westcott and T. B. Marder, Diboron(4) Compounds: From Structural Curiosity to Synthetic Workhorse, Chem. Rev., 2016, 116, 9091-9161.

5 J. Wang, W. Zheng and Y. Zheng, Theoretical study on homolytic B-B cleavages of diboron(4) compounds, RSC Adv., 2017, 7, 49251-49272.

6 M. Eck, S. Würtemberger-Pietsch, A. Eichhorn, J. H. J. Berthel, R. Bertermann, U. S. D. Paul, H. Schneider, A. Friedrich, C. Kleeberg, U. Radius and T. B. Marder, B-B bond activation and NHC ring-expansion reactions of diboron(4) compounds, and accurate molecular structures of B2(NMe2)4, B2eg2, B2neop2 and B2pin2, Dalton Trans., 2017, 46, 3661-3680. 
7 A. F. Eichhorn, L. Kuehn, T. B. Marder and U. Radius, Facile insertion of a cyclic alkyl(amino) carbene carbon into the B-B bond of diboron(4) reagents, Chem. Commun., 2017, 53, 11694-11696.

8 Y. Katsuma, H. Asakawa and M. Yamashita, Reactivity of highly Lewis acidic diborane(4) towards pyridine and isocyanide: Formation of boraalkene-pyridine complex and: Ortho -functionalized pyridine derivatives, Chem. Sci., 2018, 9, 1301-1310.

9 A. F. Pécharman, N. A. Rajabi, M. S. Hill, C. L. McMullin and M. F. Mahon, Diborane heterolysis and $\mathrm{P}(\mathrm{v})$ reduction by $\mathrm{Ph}_{3} \mathrm{P}=\mathrm{O}$ coordination to magnesium, Chem. Commun., 2019, 55, 9035-9038.

10 S. Pietsch, U. Paul, I. A. Cade, M. J. Ingleson, U. Radius and T. B. Marder, Room Temperature Ring Expansion of N-Heterocyclic Carbenes and B-B Bond Cleavage of Diboron(4) Compounds, Chem. - Eur. J., 2015, 21, 9018-9021.

11 T. Ishiyama, K. Ishida and N. Miyaura, Synthesis of pinacol arylboronates via cross-coupling reaction of bis(pinacolato)diboron with chloroarenes catalyzed by palladium(0)tricyclohexylphosphine complexes, Tetrahedron, 2001, 57, 9813-9816.

12 A. Cossaro, M. Puppin, D. Cvetko, G. Kladnik, A. Verdini, M. Coreno, M. De Simone, L. Floreano and A. Morgante, Tailoring SAM-on-SAM formation, J. Phys. Chem. Lett., 2011, 2, 3124-3129.

13 Z. Feng, G. Kladnik, G. Comelli, C. Dri and A. Cossaro, Growth of regular nanometric molecular arrays on a functional 2D template based on a chemical guest-host approach, Nanoscale, 2018, 10, 2067-2072.

14 J. V. Barth, Molecular architectonic on metal surfaces, Annu. Rev. Phys. Chem., 2007, 58, 375-407.

15 M. Stredansky, A. Sala, T. Fontanot, R. Costantini, C. Africh, G. Comelli, L. Floreano, A. Morgante and A. Cossaro, Onsurface synthesis of a $2 \mathrm{D}$ boroxine framework: a route to a novel 2D material?, Chem. Commun., 2018, 54, 3971-3973.

16 D. Toffoli, A. Ponzi, E. Bernes, M. De Simone, C. Grazioli, M. Coreno, M. Stredansky, A. Cossaro and G. Fronzoni, Correlation effects in B 1s core-excited states of boronic-acid derivatives: An experimental and computational study, J. Chem. Phys., 2019, 151, 134306.

17 J. C. Slater, in Advances in Quantum Chemistry, ed. P.-O. Löwdin, Academic Press, 1972, vol. 6, pp. 1-92.

18 L. Triguero, L. G. M. Pettersson and H. Ågren, Calculations of near-edge X-ray-absorption spectra of gas-phase and chemisorbed molecules by means of density-functional and transition-potential theory, Phys. Rev. B: Condens. Matter Mater. Phys., 1998, 58, 8097-8110.

19 W. Parr and R. G. Yang, Density-functional theory of atoms and molecules, Oxford University Press, New York, 1989.

20 C. F. Guerra, J. G. Snijders, G. te Velde and E. J. Baerends, Towards an order-N DFT method, Theor. Chem. Acc., 1998, 99, 391-403.

21 W. Clegg, M. R. J. Elsegood, F. J. Lawlor, N. C. Norman, N. L. Pickett, E. G. Robins, A. J. Scott, P. Nguyen, N. J. Taylor and T. B. Marder, Structural Studies of Bis-Catecholate, Bis-
Dithiocatecholate, and Tetraalkoxy Diborane(4) Compounds, Inorg. Chem., 1998, 37, 5289-5293.

22 J. P. Perdew, Density-functional approximation for the correlation energy of the inhomogeneous electron gas, Phys. Rev. B: Condens. Matter Mater. Phys., 1986, 33, 8822-8824.

23 J. P. Perdew, Erratum: Density-functional approximation for the correlation energy of the inhomogeneous electron gas, Phys. Rev. B: Condens. Matter Mater. Phys., 1986, 34, 7406.

24 E. J. Baerends, D. E. Ellis and P. Ros, Self-consistent molecular Hartree-Fock-Slater calculations I. The computational procedure, Chem. Phys., 1973, 2, 41-51.

25 P. Norman and A. Dreuw, Simulating X-ray Spectroscopies and Calculating Core-Excited States of Molecules, Chem. Rev., 2018, 118, 7208-7248.

26 A. D. Becke, Density-functional thermochemistry. III. The role of exact exchange, J. Chem. Phys., 1993, 98, 5648.

27 C. Lee, W. Yang and R. G. Parr, Development of the ColleSalvetti correlation-energy formula into a functional of the electron density, Phys. Rev. B: Condens. Matter Mater. Phys., 1988, 37, 785-789.

28 P. J. Stephens, F. J. Devlin, C. F. Chabalowski and M. J. Frisch, Ab Initio Calculation of Vibrational Absorption and Circular Dichroism Spectra Using Density Functional Force Fields, J. Phys. Chem., 1994, 98, 11623-11627.

29 T. Ziegler, A. Rauk and E. J. Baerends, On the calculation of multiplet energies by the Hartree-Fock-slater method, Theor. Chim. Acta, 1977, 43, 261-271.

30 P.-O. Löwdin, Quantum Theory of Many-Particle Systems. I. Physical Interpretations by Means of Density Matrices, Natural Spin-Orbitals, and Convergence Problems in the Method of Configurational Interaction, Phys. Rev., 1955, 97, 1474-1489.

31 S. Bourne Worster, O. Feighan and F. R. Manby, Reliable transition properties from excited-state mean-field calculations, J. Chem. Phys., 2021, 154, 124106.

32 K. C. Prince, R. R. Blyth, R. Delaunay, M. Zitnik, J. Krempasky, J. Slezak, R. Camilloni, L. Avaldi, M. Coreno, G. Stefani, C. Furlani, M. De Simone and S. Stranges, The gasphase photoemission beamline at Elettra, J. Synchrotron Radiat., 1998, 5, 565-568.

33 R. Costantini, M. Stredansky, D. Cvetko, G. Kladnik, A. Verdini, P. Sigalotti, F. Cilento, F. Salvador, A. De Luisa, D. Benedetti, L. Floreano, A. Morgante, A. Cossaro and M. Dell'Angela, ANCHOR-SUNDYN: A novel endstation for time resolved spectroscopy at the ALOISA beamline, J. Electron Spectrosc. Relat. Phenom., 2018, 229, 7-12.

34 I. Ljubić, M. T. Cvitaš, C. Grazioli, M. Coreno, S. Kazazić and I. Novak, Vibrationally resolved valence and core photoionization and photoexcitation spectra of an electrondeficient trivalent boron compound: The case of catecholborane, Phys. Chem. Chem. Phys., 2020, 22, 25396-25407.

35 D. Toffoli, M. Stredansky, Z. Feng, G. Balducci, S. Furlan, M. Stener, H. Ustunel, D. Cvetko, G. Kladnik, A. Morgante, A. Verdini, C. Dri, G. Comelli, G. Fronzoni and A. Cossaro, Electronic properties of the boroxine-gold interface: evidence of ultra-fast charge delocalization, Chem. Sci., 2017, 8, 3789-3798. 